\title{
Efficient cloning system for construction of gene silencing vectors in Aspergillus niger
}

\author{
José Miguel Oliveira $\cdot$ Douwe van der Veen • \\ Leo H. de Graaff • Ling Qin
}

Received: 1 April 2008 /Revised: 29 July 2008 / Accepted: 30 July 2008 / Published online: 15 August 2008

(C) The Author(s) 2008. This article is published with open access at Springerlink.com

\begin{abstract}
An approach based on Gateway recombination technology to efficiently construct silencing vectors was developed for use in the biotechnologically important fungus Aspergillus niger. The transcription activator of xylanolytic and cellulolytic genes $\mathrm{X} \operatorname{lnR}$ of $A$. niger was chosen as target for gene silencing. Silencing was based on the expression vector pXLNRir that was constructed and used in co-transformation. From all the strains isolated $(N=77)$, nine showed poor xylan-degrading activities in two semiquantitative plate assays testing different activities for xylan degradation. Upon induction on D-xylose, transcript levels of $x \ln R$ were decreased in the $x \ln R$-silenced strains, compared to a wild-type background. Under these conditions, the transcript levels of $x y r \mathrm{~A}$ and $x y n \mathrm{~B}$ (two genes regulated by $\mathrm{X} \operatorname{lnR}$ ) were also decreased for these $x \ln R$ silenced strains. These results indicate that the newly developed system for rapid generation of silencing vectors is an effective tool for A. niger, and this can be used to generate strains with a tailored spectrum of enzyme activities or product formation by silencing specific genes encoding, e.g., regulators such as $\mathrm{X} \operatorname{lnR}$.
\end{abstract}

Keywords pFIRD1 - Gateway cloning $\cdot$ Aspergillus niger . Post-transcriptional gene silencing $\cdot \mathrm{X} \ln \mathrm{R}$

\section{Introduction}

Aspergillus niger is a filamentous fungus with prominent applications in biotechnological processes. Products of the

J. M. Oliveira $\cdot$ D. van der Veen $\cdot$ L. H. de Graaff $(\bowtie) \cdot$ L. Qin Laboratory of Microbiology, Wageningen University,

Dreijenplein 10,

6703 HB Wageningen, The Netherlands

e-mail: leo.degraaff@wur.nl fungus have the generally recognized as safe (GRAS) status allowing them to be used as food additives. Important products that are made by fermentation of $A$. niger are organic acids, such as citric acid and gluconic acid. Citric acid is used for acidification and to enhance taste and flavour in food (Ruijter et al. 2000). A. niger is also an important producer of enzymes that are used in food and feed applications, such as xylanases as a bread improver and pectinases in juice clarification.

Genetic and genomic tools have been developed for this fungus over the past two decades, allowing specific modification and strain improvement to enhance the production of specific enzymes. The potential use of new genomic tools has increased with the publication of the genome sequence of $A$. niger (Pel et al. 2007). Recently, successful gene knockout strategies were carried out in strains with defective pathways for non-homologous integration (Meyer et al. 2007). However, these rely on a mutant background, and analysis of essential genes is still not straightforward, since it is limited to heterokaryon formation in the case of A. niger. Moreover, as reviewed (Meyer 2008), these engineered strains are more susceptible to toxins and irradiation, probably because $\mathrm{Ku}$ proteins have a vital role in maintenance of telomere length and chromosome stability. In addition, because this method needs a mutant background, it is not applicable toward industrial strains.

In recent years, gene silencing mediated by double-stranded (ds)RNA has opened new possibilities for the study of gene function in many organisms. Starting with pioneer studies on post-transcriptional gene silencing (PTGS) phenomena (Napoli et al. 1990; van der Krol et al. 1990; Romano and Macino 1992), many aspects of PTGS by dsRNA have been elucidated after the first study reporting the concept of dsRNA-mediated gene silencing (Fire et al. 1998).

In fungi, a number of genes involved in silencing were first described for Neurospora crassa (Cogoni and Macino 
1997) and included QDE-1 (Cogoni and Macino 1999a), QDE-2 (Catalanotto et al. 2000), QDE-3 (Cogoni and Macino 1999b) and DCL1 (sms-3)/DCL2 (Catalanotto et al. 2004). Since then, many vectors for RNA-mediated gene silencing in fungi have been used successfully (Goldoni et al. 2004; Fitzgerald et al. 2004; Mouyna et al. 2004; Nakayashiki et al. 2005). Interestingly, there is indication of occurrence of small interfering RNA-mediated mechanisms in A. niger (Barnes et al. 2008).

In some cases, the use of RNA interference (RNAi) technologies may be advantageous compared to other genetargeting techniques. "'First, for the study of essential genes since RNAi results in down-regulation of the gene function and not in loss-off-function, as is the case with a knockout strategy. Second, multiple copies of a gene or paralogous genes may be targeted with a single construct. Third, if the transformation system is based on a dominant selection marker as, e.g., the amdS gene, a method inducing gene down-regulation can be used in industrial strains.

Functional genomics based on large-scale RNAi screens has been attempted for many organisms (Berns et al. 2004; Paddison et al. 2004; Kamath et al. 2003; Boutros et al. 2004). The application of RNAi high-throughput screens to the study of gene functions in filamentous fungi, however, is still in its initial phase.

In A. niger, the expression of the biotechnologically important xylanase and cellulase enzymes is controlled by the Gal4-type transcription factor XlnR (van Peij et al. 1998b). XlnR controls not only the transcription of more than ten genes involved in the extracellular degradation of cellulose and hemi-cellulose (van Peij et al. 1998a) but also the transcription of genes that are involved in intracellular Dxylose metabolism (Hasper et al. 2000). The XlnR regulon has extensively been studied by Northern blot analysis (van Peij et al. 1998a), and many aspects of XlnR-regulated expression of target genes are known. Standardised culture and induction conditions have been developed that could serve as a basis to study the effect of gene silencing in A. niger.

In this paper, we describe a method for simple construction of dsRNA-expression vectors under the control of a fungal constitutive promoter. This method makes use of homologous recombination between a general destination vector and a specific entry clone to generate the corresponding dsRNA expression vector (Gateway). The approach has the advantage of an easy and efficient exchange of DNA fragments to produce a dsRNA-expression vector. Using this approach, we were able to target for silencing the transcriptional activator $\mathrm{X} \operatorname{lnR}$ in $A$. niger. We have analysed the effects of silencing of the transcriptional regulator towards the total expression of xylanases by functional assays and by measuring relative expression levels of two highly transcribed target genes encoding D-xylose reductase and endo- $\beta-1,4$-xylanase $B$.

\section{Materials and methods}

Fungal strains and media

A. niger strains used were derived from strain NW219 (cspA1 nicAl leuAl pyrA6), a derivative of CBS 120.49. Solid and liquid media were based on minimal medium (MM; Pontecorvo et al. 1953), contained $1 \mathrm{ml}$ of trace elements solution (Vishniac and Santer 1957) per litre of medium and were supplemented with $16 \mu \mathrm{M}$ nicotinic acid, $1.5 \mathrm{mM}$ leucine and $5.0 \mathrm{mM}$ uridine. For strains transformed by the pyrA-containing plasmid pGW635 (Goosen et al. 1989), uridine was not added to the culture medium.

\section{Construction of destination and expression vectors}

Vector pIM3710 (Benen et al. 1999) was double digested with NsiI and NotI, and a 3.6-kb fragment was isolated from agarose and blunt-ended by T4 DNA polymerase. Vector pK7GWIWG2(I) (Karimi et al. 2002) was partially digested with $E c o \mathrm{RV}$, and a $4.1-\mathrm{kb}$ fragment was isolated from agarose. The 4.1-kb fragment (with $c c d \mathrm{~B}$ genes and homologous recombination sites in inverted repeat) was inserted by ligation into the 3.6-kb fragment of pIM3710, between the pyruvate kinase (pkiA) promoter and the endo-polygalacturonase II (pgaII) terminator from A. niger. Escherichia coli strain resistant to the toxic effects of $c c d \mathrm{~B}$ gene product (One Shot ${ }^{\circledR}$ $c c d \mathrm{~B}$ Survival ${ }^{\mathrm{TM}} \mathrm{T} 1$ Phage Resistant, Invitrogen, Carlsbad, CA, USA) was used for propagation of the resulting destination vector pFIRD1. Transformation of One Shot ${ }^{\mathbb{R}} c c d \mathrm{~B}$

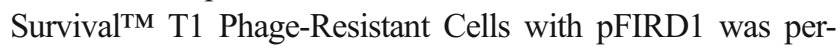
formed according to manufacturer's instructions.

The expression vector $\mathrm{pXLNRir}$ for silencing of $x \ln R$ was made as follows. A fragment of $834 \mathrm{bp}$ of $x \ln R$ coding region (plus a total of $58 \mathrm{bp}$ for att $\mathrm{B}$ recombination sites) was amplified by polymerase chain reaction (PCR) using complementary DNA (cDNA; GenBank accession no. AJ001909) isolated from A. niger NW219 as template and primers attB1$x \ln R$ and attB2-xlnR (Table 1).

The following steps were performed using Gateway technology (Invitrogen), according to manufacturer's instructions. Polyethylene glycol (PEG)/ $\mathrm{MgCl}_{2}$ purified attBproduct and pDONR201 were mixed with BP clonase II in a $10-\mu 1$ reaction mixture (BP reaction). Entry clone and destination vector pFIRD1 were mixed with LR clonase plus in a $20-\mu 1$ reaction mixture (LR reaction). In both reactions, mixtures were incubated overnight at $25^{\circ} \mathrm{C}$ and treated with proteinase $\mathrm{K}$. DH5 $\alpha$ E. coli was electrotransformed by DNA from the reaction mixture. After overnight incubation at $37^{\circ} \mathrm{C}$ on Luria-Bertani (LB) kanamycin $\left(50 \mu \mathrm{g} \cdot \mathrm{ml}^{-1}\right)$ or ampicillin $\left(100 \mu \mathrm{g} \cdot \mathrm{ml}^{-1}\right)$ plates, colonies were picked, cultured on liquid LB medium with the same antibiotic composition and incubated at $37^{\circ} \mathrm{C}$ overnight. Entry clones and expression 
Table 1 Oligonucleotide primers used for PCR in this study

\begin{tabular}{|c|c|}
\hline Primer name & Primer sequence $\left(5^{\prime}-3^{\prime}\right)$ \\
\hline attB1-xlnR & $\begin{array}{l}\text { B1-TTGAGAGCCACCATCTTAGC } \\
(\text { Bl= GGGGACAAGTTTGTACAAAAA } \\
\text { AGCAGGCT })\end{array}$ \\
\hline attB2-xlnR & $\begin{array}{l}\text { B2-TGACCATATTCGCCGCATAG } \\
\text { (B2=GGGGACCACTTTGTACAAGAAA } \\
\text { GCTGGGT) }\end{array}$ \\
\hline$x \operatorname{lnR}$-borderF & GTGAACGGCACATACGACTC \\
\hline $\mathrm{x} \operatorname{lnR}$-borderR & GAGCGACGCCATTGATGACC \\
\hline PpkiA-spacer F & GCACAGATGCGTAAGGAGAA \\
\hline PpkiA-spacer R & AGCCGTAAGAAGAGGCAAGA \\
\hline spacer-TpgaIIF & TGGTCACGCTTAGTGGGTAAA \\
\hline spacer-TpgaIIR & GGCTCGTATGTTGTGTGGAA \\
\hline IR-spacer & AGTCGCTAGCGTGTGACCTT \\
\hline$x \ln R i r-c o p y F$ & GGACTAAGCTCCGGGTACAA \\
\hline xlnRir-copyR & GAGGCGAGAGACCAAGAAAC \\
\hline pki-copyF & AACTGCTCGCTACCTTTCCA \\
\hline pki-copyR & CACGACCTCTCGAAACAACA \\
\hline$x \operatorname{lnR}$-qpcrF & GGGCAGTTTCTTGCTGCTAC \\
\hline$x \ln R-q p c r R$ & GAATGTCCTCTGGTACTCCG \\
\hline xyrA-qpcrF & TCGAGTTGAGTGTGCAGAATG \\
\hline xyrA-qpcrR & ACTCTTGGGGATAACAGCAATC \\
\hline xynB-qpcrF & CGAGTCTTACGGCGACTACA \\
\hline xynB-qpcrR & ACGGACCAGTACTGAGTGAAGG \\
\hline kan-qpcrF & AGCATTACGCTGACTTGACG \\
\hline kan-qpcrR & AGGTGGACCAGTTGGTGATT \\
\hline
\end{tabular}

vectors for RNA-mediated silencing were isolated with the Wizard Plus Minipreps DNA Purification System (Promega, Madison, WI, USA).

Fungal transformation was performed as described elsewhere (Kusters-van Someren et al. 1991). In brief, enzymatic degradation of fungal cell wall was used to generate protoplasts, followed by purification by filtration and storage in isotonic sorbitol solution. For PEG-mediated transformation, protoplasts were incubated with $1 \mu \mathrm{g}$ of pGW635 for selection and $30 \mu \mathrm{g}$ of co-transforming pXLNRir for silencing of $x \ln R$. Protoplasts transformed with $1 \mu \mathrm{g}$ of pGW635 served as control for the transformation procedure. After this procedure, protoplasts were dispersed in sucrose-stabilised MM $0.6 \%(w / v)$ agar ( $0.95 \mathrm{M}$ sucrose, $\mathrm{pH} 6.0)$ and added on sucrose-stabilised MM 1.2\% ( $w / v)$ agar with identical composition. Plates were incubated 4 days at $30^{\circ} \mathrm{C}$. After this period, spores were transferred from each strain to individual MM agar plates containing $50 \mathrm{mM}$ glucose and supplements.

Semi-quantitative assays for endoxylanase activity and $\beta$-xylosidase/xylanase activities

Spores of each isolated $A$. niger strain (transformed by pGW635 only or by pGW635 and pXLNRir) were harvested from individual MM agar plates. Saline-Tween sterile solution $[0.9 \%(w / v) \mathrm{NaCl}, 0.005 \%(v / v)$ Tween-80] was used for harvesting spores. For screening on endoxylanase activity and on $\beta$-xylosidase/xylanase activities, $5 \times 10^{4}$ and $5 \times 10^{3}$ spores of each strain were dotted on plate. Endoxylanase activity was assayed as described elsewhere (Hasper et al. 2004) on MM agar plates with $25 \mathrm{mM}$ D-xylose and $0.1 \%(w / v)$ azurine-dyed and cross-linked xylan [AZCLxylan (Megazyme, Ireland)]. $\beta$-Xylosidase/xylanase activities were assayed on MM agar plates with $0.1 \%$ oat spelts xylan (Sigma Chemical, St Louis, MO, USA) and $0.5 \mathrm{mM}$ 4-methylumbelliferyl- $\beta$-D-xylopyranoside [MUX (Sigma Chemical)]. Plates assayed for $\beta$-xylosidase/xylanase activities were incubated for $16 \mathrm{~h}$ at $30^{\circ} \mathrm{C}$, and halo formation was detected under UV light $(\lambda=280 \mathrm{~nm})$.

\section{Transfer experiments and sample quenching}

For transfer experiments, cultures were made in triplicate for each strain (transformed by pGW635 only or pGW635 and pXLNRir). Spores were inoculated at a final concentration of $10^{6}$ spores per millilitre in MM containing $0.1 \%(w / v)$ yeast extract, $0.1 \%(w / v)$ casamino acids, and $100 \mathrm{mM} \mathrm{D}$-fructose. Cultures were grown at $30^{\circ} \mathrm{C}$ in shake flasks in an orbital shaker at a constant agitation speed of $250 \mathrm{rpm}$ for $18 \mathrm{~h}$. Mycelia were subsequently harvested, transferred as described elsewhere (van Peij et al. 1998a) and grown for $4 \mathrm{~h}$ in MM with $50 \mathrm{mM}$ D-xylose for induction. After this, mycelial samples were recovered by filtration on nylon gauze, quenched in liquid nitrogen and ground in a Mikro-dismembrator II (B. Braun, Melsungen, Germany). Frozen ground mycelial samples were stored at $-80^{\circ} \mathrm{C}$ until further processing.

\section{RNA isolation and cDNA synthesis}

Frozen ground mycelial samples from transfer experiments were homogenised and lysed in Trizol Reagent (Invitrogen). Chloroform was added, and the mixtures were separated into aqueous and organic phases by phase-lock gel heavy (Eppendorf, Hamburg, Germany) according to manufacturer's instructions. The aqueous phases were transferred to an identical volume of $70 \%(v / v)$ ethanol and loaded onto RNeasy Plant Mini Kit (Qiagen, Hilden, Germany) columns for isolation and purification of RNA according to manufacturer's instructions. RNA quantity and purity was determined by spectrophotometry in a NanoDrop-1000 instrument (NanoDrop Technologies, Wilmington, DE, USA). Mixtures of $1 \mathrm{ng}$ of $1.2 \mathrm{~kb}$ kanamycin control RNA (Promega, San Luis Obispo, CA, USA) with $1 \mu \mathrm{g}$ of isolated RNA were treated with $0.1 \mathrm{U} \cdot \mu \mathrm{l}^{-1}$ DNase I (amplification grade, Invitrogen), incubated at $37^{\circ} \mathrm{C}$ for $15 \mathrm{~min}$, and DNase I was subsequently heat-inactivated according to manufacturer's recommendations. The DNase-treated RNA mixtures were used as template for Reverse Transcription reactions with Omniscript RT kit (Qiagen), using 1.0 $\mu \mathrm{M}$ Oligo(dT)18, $0.5 \mathrm{mM}$ each 
deoxyribonucleotide triphosphate, RT buffer and $0.2 \mathrm{U} \cdot \mu \mathrm{l}^{-1}$ Omniscript Reverse Transcriptase. The RT mixture was incubated for $1 \mathrm{~h}$ at $37^{\circ} \mathrm{C}$, the resulting cDNA mixture was diluted in diethyl pyrocarbonate-treated water $(1: 20)$ and stored at $-20^{\circ} \mathrm{C}$. Each cDNA sample was synthesised from each biological replicate of three independent cultures.

Quantitative real-time PCR

Primers for cDNA of $x \ln R, x y r \mathrm{~A}, x y n \mathrm{~B}$ (GenBank accession nos. AJ001909, AM269959, and AM269952) and the normaliser kanamycin external transcript (Table 1, under the label "qpcr") were designed for specific melting temperatures $\left(60^{\circ} \mathrm{C} \pm 1{ }^{\circ} \mathrm{C}\right), \mathrm{GC}$ content $(50 \% \pm 5 \%)$ and amplicon sizes $(139$ to $150 \mathrm{bp}$ ), using the program Primer3. Reagent preparation and sample pipetting for real-time PCR were performed in the CAS-1200 (Corbett Life Science, Sydney, Australia) automated system. Reaction mixtures for real-time PCR contained $25 \%(v / v)$ diluted cDNA as template (dilution, 1:20), $0.15 \mu \mathrm{M}$ each primer and ABsolute QPCR SYBR Green Mix (ABgene, Epsom, UK). Rotor-Gene 3000 (Corbett Life Science) was used for thermal cycling and real-time detection of DNA. Thermal cycling program included enzyme heat activation, amplification and melting steps. Two features of Rotor-Gene 6 software (Corbett Life Science) were used: melting analysis and comparative quantitation. The melting analysis feature was used to determine primer-dimer formation, and the comparative quantitation feature was used to determine takeoff (TO) and amplification (A) values. The estimated values were used to calculate relative gene expression levels using the Pfaffl method (Pfaffl 2001), normalised to the added kanamycin external transcript (proportional to total RNA used for cDNA synthesis) and to a standard condition (normalised expression of control A. niger NW219 transformed by pGW635 only). From each synthesised cDNA, at least three runs were considered for analysis.

\section{Genotyping by PCR}

Frozen ground mycelial samples from transfer experiments were used for isolation of genomic DNA. Genomic DNA was isolated as described elsewhere (de Graaff et al. 1988). Starting with adequate primers (Table 1) and genomic DNA as template, PCR was used to evaluate three distinct aspects of the co-transformed strains: (1) integrity of native $x \ln R$, (2) integrity of the silencing construct pXLNRir and (3) copy numbers of pXLNRir.

Integrity of native $x \ln R$ PCR with primers $x \ln R$-borderF and $\mathrm{x} \operatorname{lnR}$-borderR was used to detect native $x \ln R$. These primers were designed to anneal upstream and downstream of the 834-bp region of $x \ln R$ targeted to silencing by pXLNRir, yielding a product of $874 \mathrm{bp}$.

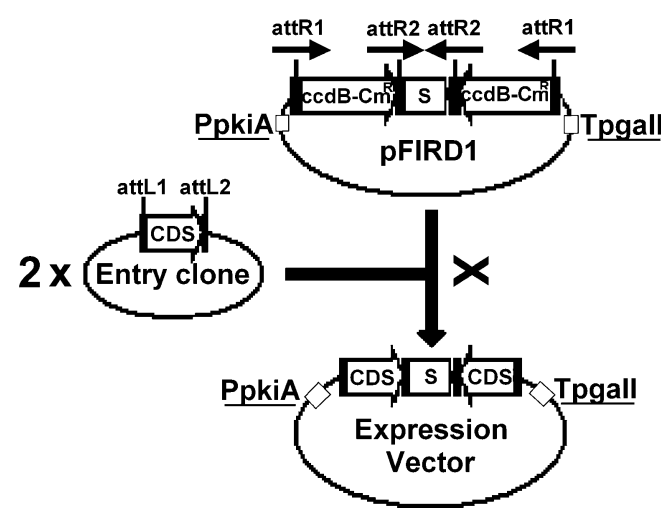

Fig. 1 Construction of expression vectors for post-transcriptional gene silencing in A. niger. The arrow indicates the recombination reaction of pFIRD1 destination vector with two molecules of entry clone containing a partial coding sequence. $c c d B-C m^{R} \operatorname{ccdB}$ genechloramphenicol resistance gene; $S$ spacer region

Integrity of $p X L N R$ ir Two regions of the silencing construct pXLNRir were targeted for PCR amplification: the upstream region spanning the pkiA promoter and part of the spacer sequence (primers used were PpkiA-spacer $\mathrm{F}$ and PpkiAspacer R) and the downstream region spanning part of the spacer sequence and the pgaII terminator (primers used were Spacer-TpgaIIF and Spacer-TpgaIIR). In addition, a portion of inverted repeat of $\mathrm{pXLNRir}$ was targeted for amplification by single-primer PCR with primer IR-spacer.

Copy numbers of $p X L N R$ ir Real-Time PCR was used to determine copy numbers of pXLNRir, with a primer pair specific for $x \ln R$ present in the inverted repeat and endogenous gene (xlnRir-copyF and $x \ln R i r-c o p y R)$ and another pair for the normaliser gene pkiA (pkiA-copyF and pkiAcopyR). Reaction mixtures for real-time PCR contained about $10 \mathrm{ng} \cdot \mu^{-1}$ genomic DNA as template, $0.15 \mu \mathrm{M}$ each primer and ABsolute QPCR SYBR Green Mix (ABgene, Epsom, UK). Thermal cycling conditions were as mentioned above for quantitative real-time PCR. For copy number

a

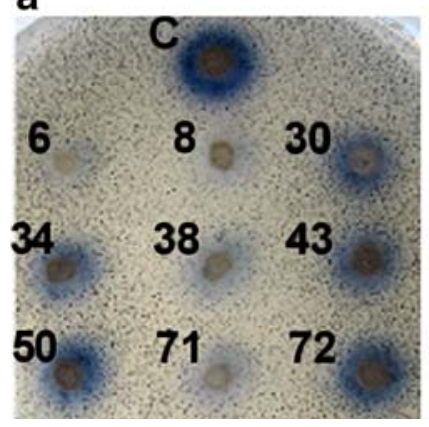

b

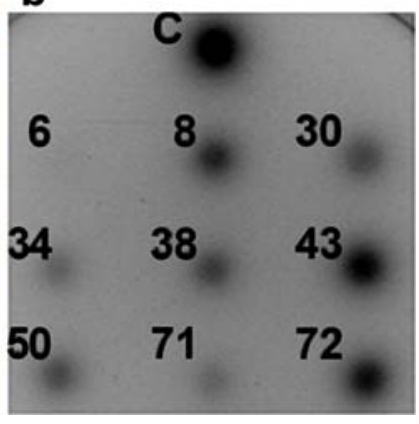

Fig. 2 Semi-quantitative assays for analysis of xylan-degrading activities. a With AZCL xylan for detection of endoxylanase activity. b With MUX for detection of $\beta$-xylosidase and xylanase activities. $C$ Wild-type strain (NW219::pGW635); numerals designate each silenced strain (NW219::pGW635::pXLNRir) 


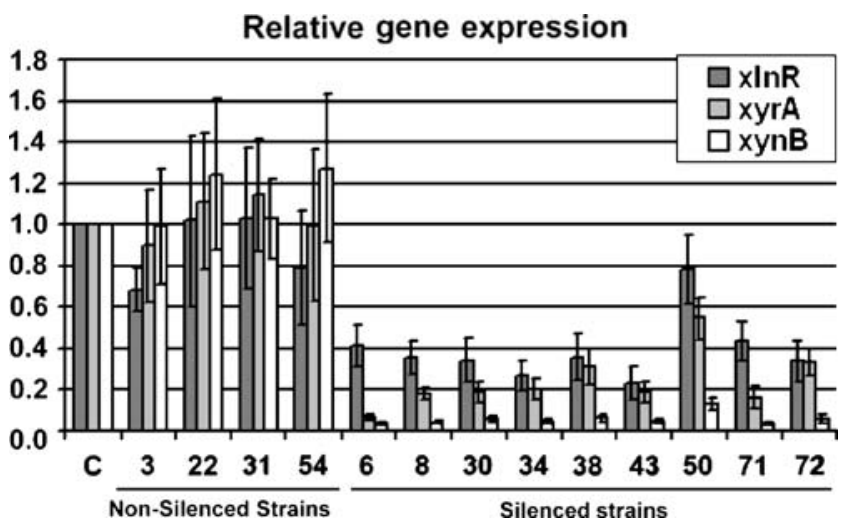

Fig. 3 mRNA levels for $x \ln R, x y r \mathrm{~A}$ and $x y n \mathrm{~B}$ in silenced and nonsilenced strains, compared to wild type. $C$ Wild-type strain (NW219:: pGW635); numerals designate each strain from co-transformation (NW219::pGW635::pXLNRir). Error bars represent 95\% confidence intervals $(1.962 \times \mathrm{SE})$

determination, the Pfaffl method (Pfaffl 2001) was used: Samples were first normalised for genomic DNA quantity using the control pkiA gene in order to determine the total number of copies of $x \ln R$ segments per genome. Afterwards, the number of $x \ln R$ segments was subtracted by one unit (one endogenous $x \ln R$ gene per genome) and divided by two (assuming that inverted repeats are integrated "en bloc" and without suffering rearrangements) to obtain the average number of inverted repeats per genome of each strain.

\section{Results}

Effect of pXLNRir on xylan-degrading activities of $A$. niger

A. niger strain NW219 was transformed by pXLNRir (under the control of the constitutive pkiA promoter and pgaII ter- minator) and by pGW635, and strains were screened for xylan-degrading activities. The silencing vector $p X L N R i r$ was constructed from the reaction between the general destination vector pFIRD1 and an appropriate entry clone containing a portion of $x \ln R$ coding sequence (CDS) as depicted (Fig. 1). After transformation, 77 colonies were obtained with no visible differences in morphology or growth rate on complete medium (compared to non-transformed NW219 or NW219 transformed only with pGW635). These strains and one control strain (NW219::pGW635) were isolated and analysed for endoxylanase activity and $\beta$ xylosidase/xylanase activities. Under these conditions, a gradient of effects was observed in halo size and intensities of colour or fluorescence resulting from substrate degradation (Fig. 2). Compared to the control strain, nine strains (12\% of 77) showed decreased activities in both assays, whereas three different strains showed decreased endoxylanase activity but no decreased $\beta$-xylosidase/xylanase activities, and one other strain showed decreased $\beta$-xylosidase/xylanase activities but no decreased endoxylanase activity. The nine strains that showed decreased activities in both assays were considered silenced transformant strains (either fully or partially silenced) and were further investigated, together with four non-silenced strains from co-transformation.

Expression of $x \ln R, x y r \mathrm{~A}$ and $x y n \mathrm{~B}$ in silenced strains

Quantitative real-time PCR was used to determine expression of $x \ln R, x y r \mathrm{~A}$ and $x y n \mathrm{~B}$ in the silenced strains and in four non-silenced strains relative to the control strain (NW219:: pGW635). All samples were first normalised to the added kanamycin external transcript, directly proportional to the amount of total RNA. As a second normalisation step, gene expression relative to the control strain was calculated for each strain. Strain 50 was an outlier because, compared to
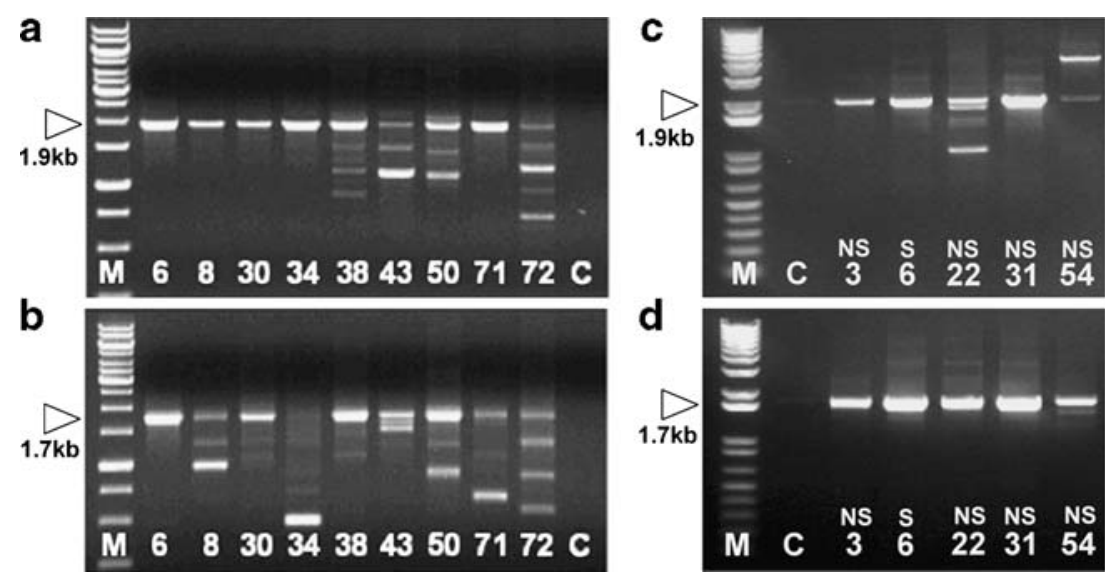

Fig. 4 Genome integrity of pXLNRir. Genome integrity was assessed for both silenced (a and b) and non-silenced strains (c and d). a, c PCR-amplified 5'-region of pXLNRir. b, d PCR-amplified 3'-region of pXLNRir. Each triangle represents the expected fragment for intact

pXLNRir. $M$ Molecular weight marker; numerals designate each strain from co-transformation (NW219::pGW635::pXLNRir); $C$ wild-type strain (NW219::pGW635) 


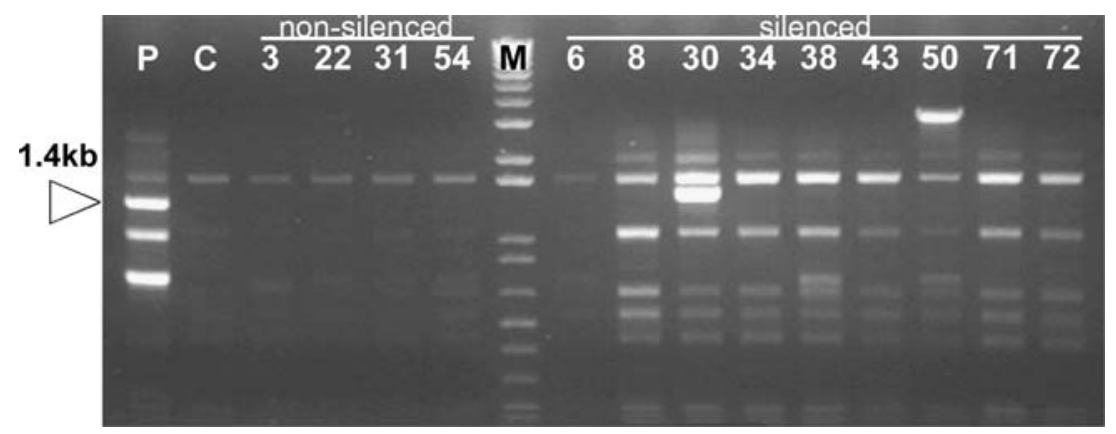

Fig. 5 Inverted repeats of pXLNRir. PCR-amplified portion of inverted repeat of $\mathrm{pXLNRir}$ from genomic DNA of non-silenced and silenced strains. Triangle Expected fragment for intact inverted repeat

the group of silenced strains, it showed significantly higher expression for all genes tested although still lower than the control strain (Fig. 3). In all silenced strains, relative gene expression was significantly decreased not only for the target gene $x \ln R$ but also for the regulated genes $x y r \mathrm{~A}$ and $x y n \mathrm{~B}$. Moreover, for each silenced strain, a moderate decrease in $x \ln R$ levels (to about $25-40 \%$ of wild-type expression levels) was associated with a larger decrease in $x y r \mathrm{~A}$ and $x y n \mathrm{~B}$ expression levels (about 15\% and 10\% of wild-type expression levels, respectively). As expected, expression levels for $x \ln R, x y r \mathrm{~A}$ and $x y n \mathrm{~B}$ in the non-silenced strains were similar to wild-type levels within the experimental error.

Genome rearrangements and copy numbers of $\mathrm{pXLNRir}$ in silenced strains

Two approaches were taken for genotypic characterisation of all silenced strains: a qualitative approach based on conventional PCR and a more quantitative approach based on quantitative real-time PCR.

Through conventional PCR a fragment of about 874 bp of native $x \ln R$ was found in all strains using primers binding to $x \ln R$ but not to pXLNRir, indicating that, in each case, the endogenous $x \ln R$ locus was not affected (data not shown). Moreover, to detect genome rearrangements within integrated pXLNRir, two fragments covering the hairpin RNA expression cassette were amplified separately by PCR. Rearrangements were detected in all silenced strains (Fig. 4), although strains 6 and 30 mostly retained the two parts of the original construct according to this analysis. Strain 34 in contrast carried a truncated version of the hairpin RNA expression cassette at the 3'-region. The non-silenced strains also showed amplification of both fragments spanning the inverted repeat of pXLNRir (Fig. 4). PCR amplification of part of the inverted repeat region of $\mathrm{pXLNRir}$ followed by agarose gel electrophoresis showed that, within the group of silenced strains, a larger variety of fragments with stronger band intensity were produced compared to non-silenced strains (Fig. 5). Strain 6 was an outlier because an amplification profile more region. $M$ Molecular weight marker; $P$ silencing plasmid pXLNRir; $C$ wild-type strain (NW219::pGW635); numerals designate each nonsilenced and silenced strain (NW219::pGW635::pXLNRir)

similar to that of non-silenced strains was obtained. In addition, specific amplification product was only obtained for strain 30. Furthermore, aspecific amplification was observed for the wild-type control strain (data not shown).

For quantitative analysis, a portion of $x \ln \mathrm{R}$ coding sequence present both in native $x \ln \mathrm{R}$ and in $\mathrm{pXLNRir}$ was amplified and detected by real-time PCR. After normalization, diverse copy numbers were obtained, indicating variable frequencies of integration of pXLNRir in the different silenced strains (Table 2).

\section{Discussion}

Phenotypic screens are widely used for strain improvement in industry. For most industrial strains, a desirable phenotype

Table 2 Copy numbers of pXLNRir in control strain C (NW219:: pGW635), non-silenced and silenced strains (NW219::pGW635:: pXLNRir)

\begin{tabular}{ll}
\hline Strains & Estimated copy numbers of pXLNRir \\
\hline Control & \\
C & 0 \\
Non-silenced & 1 \\
Strain 3 & 2 to 3 \\
Strain 22 & 10 to 16 \\
Strain 31 & 0 to 1 \\
Strain 54 & 3 or 4 \\
Silenced & 1 or 2 \\
Strain 6 & 2 \\
Strain 8 & 5 or 6 \\
Strain 30 & 5 to 7 \\
Strain 34 & 1 to 2 \\
Strain 38 & 9 to 13 \\
Strain 43 & 2 \\
Strain 50 & 4 to 9 \\
Strain 71 & \\
Strain 72 &
\end{tabular}

Estimated copy numbers varied according to the standard deviation of measurements from three quantitative real-time-PCR runs. 
such as increased or decreased enzyme production can be readily detected through simple functional assays. The system reported in this paper relies on downstream screening of easily characterisable phenotypes. Alternatively, in case a clear phenotypic difference cannot be obtained by plate screening of isolates, reverse-transcription PCR or Northern blot analysis may be used to confirm silencing. The Gatewaybased technology presented in this study is based on exchange of fragments by means of two recombination reactions (BP and LR reactions). This recombination technology has the advantage that fragments originating from different genes can efficiently be inserted into an appropriate destination vector such as pFIRD1. For the construction of pXLNRir, a stem length of 834 nucleotides of $x \ln R$ coding sequence was used, in accordance to a previous finding that stem lengths of 600 or 900 nucleotides displayed higher silencing efficiencies in the ascomycete $N$. crassa (Goldoni et al. 2004).

After transformation of $A$. niger by pXLNRir, a gradient of effects was observed on xylan-degrading activities. Although the strains with decreased xylanolytic activity obtained presented a moderately efficient silencing degree (except for strain 50, about $30 \%$ the $x \ln R$ expression of wildtype $A$. niger), the number of silenced strains in comparison to the total number of strains obtained by co-transformation (12\% of the total) was lower than expected. A number of reasons could serve as explanation for this result. First, some transformants might not be true co-transformants, even though this was not the case for the four non-silenced strains analysed. Second, some co-transformants such as nonsilenced strain 54 suffered rearrangements in silencing construct. Third, silencing of $x \ln \mathrm{R}$ was not significant in all cases to yield an obvious decrease of xylanolytic activities, such as in the case of non-silenced strains 3 and 54 .

Two confounding factors in the analysis of RNAi transformants are genome rearrangements, which may occur after fungal transformation with silencing vectors and alternate silencing pathways not involving a typical Dicer-like/RISCmediated degradation of mRNA (e.g. chromatin remodelling, DNA methylation and repeat induced mutation). In fact, nonsilenced transformants often carry full silencing vectors, and silenced transformants often carry only altered versions of hairpin-expression units, as previously determined (Fitzgerald et al. 2004; Moriwaki et al. 2007; Nakayashiki et al. 2005). The separate analysis of either agarose gels of amplified regions of pXLNRir from genomic DNA or of estimated copy numbers of integrated pXLNRir is not conclusive. On the one hand, rearrangements may affect copy number determinations (even using Southern blotting techniques, which frequently miss smaller variations), and on the other hand, agarose gel analysis of amplified segments of genomic DNA is insufficient to provide reliable quantification. Nevertheless, combined analysis of these two methods may be used: Fr example, copy numbers of pXLNRir do not correlate with a higher degree of silencing efficiency, a situation similar to others reported (Nakayashiki et al. 2005; Nicolas et al. 2003; Tanguay et al. 2006; Fitzgerald et al. 2004; Henry et al. 2007; Yamada et al. 2007; Walti et al. 2006). Apart from genome instability and the occurrence of rearrangements, other factors may be responsible for a lower silencing efficiency of integrated hairpin-expression units. These factors include integration in regions of very low transcriptional activities (such as heterochromatin regions) or epigenetic modification of the inverted repeats without co-alteration of endogenous $x \ln R$ (possibly through RNA-induced transcriptional silencing). Due to the high genetic diversity and genome instability of strains transformed with dsRNAexpression vectors, it may be of advantage to pre-select silenced transformants for single-locus integration of silencing vector, as these transformants would be less likely to undergo genome rearrangements in successive generations.

In spite of the fact that strains were obtained with varying genotypes for integration of pXLNRir, it was possible to obtain a clear silencing effect both on enzyme xylan-degrading activities and on transcript levels of the genes $x y r \mathrm{~A}$ (encoding D-xylose reductase) and $x y n \mathrm{~B}$ (also known as $x \ln \mathrm{B}$, encoding endo- $\beta$-1,4-xylanase B), previously shown to be positively regulated by $X \operatorname{lnR}$ in A. niger (Hasper et al. 2000).

In conclusion, the Gateway-based system presented in this paper has three noteworthy features: first, fast production of expression clones (about 1 week); second, use of any appropriate entry clone and third, no generation of unproductive clones. As shown for the transformation of the industrial microorganism $A$. niger with pXLNRir, it is furthermore possible to silence transcriptional regulators and screen for strains with the most desirable properties, depending on the effects of silencing on the expression of regulated genes.

Acknowledgements This research was funded by the Kluyver Centre for Genomics of Industrial Fermentation, which is supported by the Netherlands Genomics Initiative.

Open Access This article is distributed under the terms of the Creative Commons Attribution Noncommercial License which permits any noncommercial use, distribution, and reproduction in any medium, provided the original author(s) and source are credited.

\section{References}

Barnes SE, Alcocer MJC, Archer DB (2008) siRNA as a molecular tool for use in Aspergillus niger. Biotechnology Letters 30:885-890

Benen JA, Kester HC, Visser J (1999) Kinetic characterization of Aspergillus niger N400 endopolygalacturonases I, II and C. Eur J Biochem 259:577-585

Berns K, Hijmans EM, Mullenders J, Brummelkamp TR, Velds A, Heimerikx M, Kerkhoven RM, Madiredjo M, Nijkamp W, Weigelt B, Agami R, Ge W, Cavet G, Linsley PS, Beijersbergen RL, Bernards R (2004) A large-scale RNAi screen in human cells identifies new components of the p53 pathway. Nature 428:431-437 
Boutros M, Kiger AA, Armknecht S, Kerr K, Hild M, Koch B, Haas SA, Paro R, Perrimon N (2004) Genome-wide RNAi analysis of growth and viability in Drosophila cells. Science 303:832-835

Catalanotto C, Azzalin G, Macino G, Cogoni C (2000) Gene silencing in worms and fungi. Nature 404:245

Catalanotto C, Pallotta M, ReFalo P, Sachs MS, Vayssie L, Macino G, Cogoni C (2004) Redundancy of the two dicer genes in transgene-induced posttranscriptional gene silencing in Neurospora crassa. Mol Cell Biol 24:2536-2545

Cogoni C, Macino G (1997) Isolation of quelling-defective (qde) mutants impaired in posttranscriptional transgene-induced gene silencing in Neurospora crassa. Proc Natl Acad Sci U S A 94: 10233-10238

Cogoni C, Macino G (1999a) Gene silencing in Neurospora crassa requires a protein homologous to RNA-dependent RNA polymerase. Nature 399:166-169

Cogoni C, Macino G (1999b) Posttranscriptional gene silencing in Neurospora by a RecQ DNA helicase. Science 286:2342-2344

de Graaff L, van den Broek H, Visser J (1988) Isolation and transformation of the pyruvate kinase gene of Aspergillus nidulans. Curr Genet 13:315-321

Fire A, Xu S, Montgomery MK, Kostas SA, Driver SE, Mello CC (1998) Potent and specific genetic interference by doublestranded RNA in Caenorhabditis elegans. Nature 391:806-811

Fitzgerald A, Van Kan JA, Plummer KM (2004) Simultaneous silencing of multiple genes in the apple scab fungus, Venturia inaequalis, by expression of RNA with chimeric inverted repeats. Fungal Genet Biol 41:963-971

Goldoni M, Azzalin G, Macino G, Cogoni C (2004) Efficient gene silencing by expression of double stranded RNA in Neurospora crassa. Fungal Genet Biol 41:1016-1024

Goosen T, van Engelenburg F, Debets F, Swart K, Bos K, van den Broek H (1989) Tryptophan auxotrophic mutants in Aspergillus niger: inactivation of the trpC gene by cotransformation mutagenesis. Mol Gen Genet 219:282-288

Hasper AA, Visser J, de Graaff LH (2000) The Aspergillus niger transcriptional activator $\mathrm{X} \ln \mathrm{R}$, which is involved in the degradation of the polysaccharides xylan and cellulose, also regulates Dxylose reductase gene expression. Mol Microbiol 36:193-200

Hasper AA, Trindade LM, van der Veen D, van Ooyen AJ, de Graaff LH (2004) Functional analysis of the transcriptional activator XlnR from Aspergillus niger. Microbiology 150:1367-1375

Henry C, Mouyna I, Latge JP (2007) Testing the efficacy of RNA interference constructs in Aspergillus fumigatus. Curr Genet 51:277284

Kamath RS, Fraser AG, Dong Y, Poulin G, Durbin R, Gotta M, Kanapin A, Le Bot N, Moreno S, Sohrmann M, Welchman DP, Zipperlen P, Ahringer J (2003) Systematic functional analysis of the Caenorhabditis elegans genome using RNAi. Nature 421:231-237

Karimi M, Inze D, Depicker A (2002) GATEWAY vectors for Agrobacterium-mediated plant transformation. Trends Plant Sci 7:193-195

Kusters-van Someren MA, Harmsen JA, Kester HC, Visser J (1991) Structure of the Aspergillus niger pelA gene and its expression in Aspergillus niger and Aspergillus nidulans. Curr Genet 20:293299

Meyer V (2008) Genetic engineering of filamentous fungi-progress, obstacles and future trends. Biotechnol Adv 26:177-185

Meyer V, Arentshorst M, El-Ghezal A, Drews AC, Kooistra R, van den Hondel CA, Ram AF (2007) Highly efficient gene targeting in the Aspergillus niger kusA mutant. J Biotechnol 128:770-775

Moriwaki A, Ueno M, Arase S, Kihara J (2007) RNA-mediated gene silencing in the phytopathogenic fungus Bipolaris oryzae. FEMS Microbiology Lett 269:85-89
Mouyna I, Henry C, Doering TL, Latge JP (2004) Gene silencing with RNA interference in the human pathogenic fungus Aspergillus fumigatus. FEMS Microbiol Lett 237:317-324

Nakayashiki H, Hanada S, Nguyen BQ, Kadotani N, Tosa Y, Mayama S (2005) RNA silencing as a tool for exploring gene function in ascomycete fungi. Fungal Genet Biol 42:275-283

Napoli C, Lemieux C, Jorgensen R (1990) Introduction of a chimeric chalcone synthase gene into petunia results in reversible cosuppression of homologous genes in trans. Plant Cell 2:279-289

Nicolas FE, Torres-Martinez S, Ruiz-Vazquez RM (2003) Two classes of small antisense RNAs in fungal RNA silencing triggered by non-integrative transgenes. Embo J 22:3983-3991

Paddison PJ, Silva JM, Conklin DS, Schlabach M, Li M, Aruleba S, Balija V, O'Shaughnessy A, Gnoj L, Scobie K, Chang K, Westbrook T, Cleary M, Sachidanandam R, McCombie WR, Elledge SJ, Hannon GJ (2004) A resource for large-scale RNAinterference-based screens in mammals. Nature 428:427-431

Pel HJ, de Winde JH, Archer DB, Dyer PS, Hofmann G, Schaap PJ, Turner G, de Vries RP, Albang R, Albermann K, Andersen MR, Bendtsen JD, Benen JA, van den Berg M, Breestraat S, Caddick MX, Contreras R, Cornell M, Coutinho PM, Danchin EG, Debets AJ, Dekker P, van Dijck PW, van Dijk A, Dijkhuizen L, Driessen AJ, d'Enfert C, Geysens S, Goosen C, Groot GS, de Groot PW, Guillemette T, Henrissat B, Herweijer M, van den Hombergh JP, van den Hondel CA, van der Heijden RT, van der Kaaij RM, Klis FM, Kools HJ, Kubicek CP, van Kuyk PA, Lauber J, Lu X, van der Maarel MJ, Meulenberg R, Menke H, Mortimer MA, Nielsen J, Oliver SG, Olsthoorn M, Pal K, van Peij NN, Ram AF, Rinas U, Roubos JA, Sagt CM, Schmoll M, Sun J, Ussery D, Varga J, Vervecken W, van de Vondervoort PJ, Wedler H, Wosten HA, Zeng AP, van Ooyen AJ, Visser J, Stam H (2007) Genome sequencing and analysis of the versatile cell factory Aspergillus niger CBS 513.88. Nat Biotechnol 25:221-231

Pfaffl MW (2001) A new mathematical model for relative quantification in real-time RT-PCR. Nucleic Acids Res 29:e45

Pontecorvo G, Roper JA, Hemmons LM, Macdonald KD, Bufton AW (1953) The genetics of Aspergillus nidulans. Adv Genet 5:141-238

Romano N, Macino G (1992) Quelling: transient inactivation of gene expression in Neurospora crassa by transformation with homologous sequences. Mol Microbiol 6:3343-3353

Ruijter GJ, Panneman H, Xu D, Visser J (2000) Properties of Aspergillus niger citrate synthase and effects of citA overexpression on citric acid production. FEMS Microbiol Lett 184:35-40

Tanguay P, Bozza S, Breuil C (2006) Assessing RNAi frequency and efficiency in Ophiostoma floccosum and O. piceae. Fungal Genet Biol 43:804-812

van der Krol AR, Mur LA, Beld M, Mol JN, Stuitje AR (1990) Flavonoid genes in petunia: addition of a limited number of gene copies may lead to a suppression of gene expression. Plant Cell 2:291-299

van Peij NN, Gielkens MM, de Vries RP, Visser J, de Graaff LH (1998a) The transcriptional activator $\mathrm{X} \ln \mathrm{R}$ regulates both xylanolytic and endoglucanase gene expression in Aspergillus niger. Appl Environ Microbiol 64:3615-3619

van Peij NN, Visser J, de Graaff LH (1998b) Isolation and analysis of $\mathrm{x} \operatorname{lnR}$, encoding a transcriptional activator co-ordinating xylanolytic expression in Aspergillus niger. Mol Microbiol 27:131-142

Vishniac W, Santer M (1957) The thiobacilli. Bacteriol Rev 21:195-213

Walti MA, Villalba C, Buser RM, Grunler A, Aebi M, Kunzler M (2006) Targeted gene silencing in the model mushroom Coprinopsis cinerea (Coprinus cinereus) by expression of homologous hairpin RNAs. Eukaryot Cell 5:732-744

Yamada O, Ikeda R, Ohkita Y, Hayashi R, Sakamoto K, Akita O (2007) Gene silencing by RNA interference in the koji mold Aspergillus oryzae. Biosci Biotechnol Biochem 71:138-144 